A New Stable Splitting for the Isentropic Euler Equations

Peer-reviewed author version

KAISER, Klaus; SCHUETZ, Jochen; Schöbel, Ruth \& Noelle, Sebastian (2017) A

New Stable Splitting for the Isentropic Euler Equations. In: Journal of scientific computing, 70(3), p. 1390-1407.

DOI: $10.1007 / \mathrm{s} 10915-016-0286-6$

Handle: http://hdl.handle.net/1942/22026 


\title{
A new stable splitting for the isentropic Euler equations
}

\author{
Klaus Kaiser • Jochen Schütz • Ruth \\ Schöbel · Sebastian Noelle
}

Received: date / Accepted: date

\begin{abstract}
In this work, we propose a new way of splitting the flux function of the isentropic compressible Euler equations at low Mach number into stiff and non-stiff parts. Following the IMEX methodology, the latter ones are treated explicitly, while the first ones are treated implicitly. The splitting is based on the incompressible limit solution, which we call reference solution (RS). An analysis concerning the asymptotic consistency and numerical results demonstrate the advantages of this splitting.

Keywords: flux splitting, isentropic Euler equations, low Mach, IMEX, reference solution
\end{abstract}

AMS subject classification: 35L65, 76N, 76M45, 65M08

\section{Introduction}

In this paper, we propose a particularly stable and accurate flux-splitting IMEX scheme for stiff systems of hyperbolic conservation laws. We derive, analyze and evaluate the scheme for low Mach number flows governed by the isentropic compressible Euler equations in two spatial dimensions (see, e.g., $[1,44])$.

K. Kaiser* and S. Noelle

Institut für Geometrie und Praktische Mathematik, RWTH Aachen University

Templergraben 55, 52062 Aachen, Germany

E-mail: kaiser@igpm.rwth-aachen.de

J. Schütz

Faculty of Sciences, Hasselt University, Campus Diepenbeek

Agoralaan Gebouw D, 3590 Diepenbeek, Belgium

R. Schöbel

Institute for Advanced Simulation, Forschungszentrum Jülich GmbH

Wilhelm-Jonen-Straße, 52425 Jülich, Germany 
The isentropic compressible Euler equations, written in dimensionless variables, are given by

$$
\begin{array}{r}
\rho_{t}+\nabla \cdot(\rho \mathbf{u})=0 \\
(\rho \mathbf{u})_{t}+\nabla \cdot(\rho \mathbf{u} \otimes \mathbf{u})+\frac{1}{\varepsilon^{2}} \nabla p=0
\end{array}
$$

where $\mathbf{u}=(u, v)^{T}$ denotes velocity, $\rho$ density and $p \equiv p(\rho)=\rho^{\gamma}$ is the isentropic pressure. The constant $\gamma \geq 1$ is the ratio of specific heats. We call

$$
\varepsilon:=\frac{u_{\mathrm{ref}}}{\sqrt{p\left(\rho_{\mathrm{ref}}\right) / \rho_{\mathrm{ref}}}}
$$

the reference Mach number, with fixed dimensional reference speed $u_{\text {ref }}$ and density $\rho_{\text {ref. }}$ The system is hyperbolic with wave speeds

$$
\lambda_{0}=\mathbf{u} \cdot \mathbf{n}, \quad \lambda_{ \pm}=\mathbf{u} \cdot \mathbf{n} \pm \frac{1}{\varepsilon} \sqrt{\frac{\gamma p}{\rho}}
$$

in direction $\mathbf{n}$. We are interested in weakly compressible flows, for which $\varepsilon \ll 1$. It is well-known that the equations change type as $\varepsilon \rightarrow 0$. Physically, the flow changes from compressible to incompressible. It is instructive to consider the Hilbert expansion (often also named after Poincaré) of $\mathbf{w}=(\rho, \rho \mathbf{u})^{T}$, i.e.,

$$
\mathbf{w}=\mathbf{w}_{(0)}+\varepsilon \mathbf{w}_{(1)}+\varepsilon^{2} \mathbf{w}_{(2)}+\ldots
$$

Taking the formal limit $\varepsilon \rightarrow 0$ of the isentropic compressible Euler equation, one derives the incompressible equations as

$$
\begin{aligned}
& \rho_{(0)} \equiv \text { const, } \quad \nabla \cdot \mathbf{u}_{(0)}=0 \\
& \left(\mathbf{u}_{(0)}\right)_{t}+\left(\mathbf{u}_{(0)} \cdot \nabla\right) \mathbf{u}_{(0)}+\frac{1}{\rho_{(0)}} \nabla p_{(2)}=0 .
\end{aligned}
$$

Note that one can only obtain the limit if one uses well-prepared initial data $\mathbf{w}(t=0)$, i.e., initial data that fulfill

$$
\rho(t=0)=\mathrm{const}+\mathcal{O}\left(\varepsilon^{2}\right), \quad \nabla \cdot \mathbf{u}(t=0)=\mathcal{O}(\varepsilon) .
$$

Mathematically, the system changes from hyperbolic to hyperbolic-elliptic (see, e.g., [31,40,46]). Therefore, (1)-(2) constitute a singularly perturbed problem. For a mathematical analysis of this problem we refer to $[31,40,46]$ and the references therein. For conciseness, we write equations (1)-(2) as a general hyperbolic system of conservation laws

$$
\mathbf{w}_{t}+\nabla \cdot \mathbf{F}(\mathbf{w})=0
$$

with $\varepsilon$-dependent flux $\mathbf{F}(\mathbf{w})$ and a maximum wave speed which is singular in the limit.

Explicit time discretizations have to obey the CFL condition

$$
\Delta t \lesssim \varepsilon \Delta x
$$


For the Euler equations, the fastest waves are the sound waves, travelling with speed $\lambda_{ \pm}$. Hence we call condition (9) the acoustic CFL condition. Consequently, explicit finite volume discretizations are prohibitively expensive for small Mach numbers. Moreover, they would add an unwanted amount of numerical diffusion to the advective waves, which travel with speed $\lambda_{0}$ and hence need $\mathcal{O}\left(\varepsilon^{-1}\right)$ steps to travel across one cell - and after each step the finite volume average damps the numerical solution. It is well-known that most $\mathrm{im}$ plicit schemes are unconditionally stable, i.e., they are stable under any time step size. However, they also tend to add excessive diffusion to the advective field [33] within each single time step. A possible remedy is a mixture of both implicit and explicit (IMEX) schemes $[3,18,26]$. More precisely, those schemes are supposed to be stable under a convective CFL condition

$$
\Delta t \lesssim \frac{\Delta x}{\|\mathbf{u}\|},
$$

and the goal is to resolve the convective wave (the one that belongs to $\lambda_{0}$ ) explicitly with good accuracy, while adding enough diffusion through the implicitness to compute solutions to the acoustic waves in a stable way. For this, it is necessary that the scheme preserves the asymptotic limit.

IMEX schemes depend on a splitting of the flux $\mathbf{F}(\mathbf{w})$ into 'fast' and 'slow' components $\tilde{\mathbf{F}}(\mathbf{w})$ and $\hat{\mathbf{F}}(\mathbf{w})$, respectively. Flux splittings have been applied successfully to compressible fluid flows (see, e.g., $[8,17,20,22,24,32,35,36,38$, 45]). The fast flux component is discretized implicitly in time, while the slow component is treated explicitly, both with an $\varepsilon$-uniform time step. So ideally, $\tilde{\mathbf{F}}$ approximates the acoustic and $\hat{\mathbf{F}}$ the convective waves.

Recently, the authors and collaborators have conducted a number of numerical experiments with well-known splittings, as well as some AP analysis, reported in $[8,36]$. While the Giraldo-Restelli splitting [23] used in [8] was uniformly CFL stable in our computations, Klein's auxiliary splitting [32] occasionally requires to reduce the time step as $\Delta t=\varepsilon \Delta x$ (see [36]). With a note of caution, however, we would like to point out that the implementations and the asymptotic settings in [32] and [36] differ considerably, so our observation applies only to the straightforward IMEX implementation, and not to Klein's original algorithm.

In [43], we started a linear stability analysis based on the modified equation. It turns out that for any system of hyperbolic conservation laws, and any flux splitting, the numerical diffusion matrix of the modified equation is of the form

$$
\frac{\Delta t}{2}\left(\frac{(\widetilde{\alpha}+\widehat{\alpha}) \Delta x}{\Delta t} I-\widehat{A}^{2}+[\widetilde{A}, \widehat{A}]+\widetilde{A}^{2}\right),
$$

where $\widetilde{\alpha}$ and $\widehat{\alpha}$ are numerical diffusions coefficients of the Rusanov flux, and $A=\widetilde{A}+\widehat{A}$ are the Jacobian matrices of the full, fast and slow fluxes, respectively. $[\widetilde{A}, \widehat{A}]:=\widetilde{A} \widehat{A}-\widetilde{A} \widehat{A}$ is the commutator of the fast and slow Jacobians. We now assume that the spectral radius of the fast Jacobian is $\mathcal{O}\left(\varepsilon^{-1}\right)$, and that of the slow Jacobian is $\mathcal{O}(1)$. Then the spectral radius of $\widehat{A}^{2}$ is $\mathcal{O}(1)$ 
as well, and thus the explicit part of the algorithm can be balanced by an $\varepsilon$-uniform advective CFL condition. The fast term is $\mathcal{O}\left(\varepsilon^{-2}\right)$, which is singular, but positive (semi-)definite. Thus, the implicit part of the algorithm is unconditionally stable. The remaining suspect of causing a non-uniform CFL condition is the commutator. It may lead to a subtle resonance between the implicit and explicit parts of the algorithm. In general, it is of $\mathcal{O}\left(\varepsilon^{-1}\right)$. Hence it may be singular, and it has no sign which would rescue stability. In [43], we removed the commutator via a characteristic splitting and therefore obtained $\varepsilon$-uniform stability. This approach cannot be carried over directly to nonlinear, and to multidimensional, systems.

To overcome this difficulty, we propose a general splitting, which is linear in the implicit part, based on a reference solution and call this splitting RS-IMEX [41]. In the present paper, we choose the zeroth-order asymptotic component $\mathbf{w}_{(0)}$ as reference solution. Due to this choice, the eigenvectors of the one-dimensional stiff and non-stiff Jacobians are asymptotically close to each other (see Remark 3 below). Therefore, the commutator of stiff and non-stiff Jacobians disappears in the limit, which serves as a motivation for considering the proposed splitting.

The RS-IMEX approach was first analyzed in [41] for a singularly perturbed system of ODEs, and gave superior results for high order IMEX Runge-Kutta and BDF time discretizations. Furthermore, in [47] Zakerzadeh et al. proved stability of the modified equation for the one-dimensional shallow water equations with flat bottom. In [21], a similar splitting idea is used in the context of kinetic equations and in [22] for the pressure gradient in the shallow water equations.

The present paper develops the RS-IMEX approach for the two dimensional isentropic Euler equations, by coupling it with a first order time and space discretization. We prove the asymptotic preserving property (AP-property) [19, $27,28]$, which is fundamental in the low Mach regime, see exemplarily $[8,20$, $24,36]$ and the references therein. Roughly speaking, the AP property guarantees that the limiting numerical method (limit w.r.t. $\varepsilon \rightarrow 0$ ) is a consistent discretization of the limiting equation for fixed values of $\Delta t$ and $\Delta x$. This concept is not restricted to low Mach flows, but it can also be applied to other discretizations of singular problems. Examples are the discretization of kinetic equations, see, e.g., $[11,29,34,37]$ and the references therein, or the discretization of singularly perturbed ODEs, see, e.g., $[9,10,25,41]$ and the references therein. This list is by no means exhaustive, we refer to [28] for a more detailed overview.

We compare our scheme to a first order IMEX scheme with a well-established flux splitting due to Haack, Jin and Liu [24]. Our numerical tests show a considerable advantage of the RS-IMEX scheme for small Mach numbers, both in terms of error and computing time.

The paper is organized as follows: The splitting is defined in more detail in the second section; in the third section, we discuss the asymptotic preserving property. In the fourth section, numerical results underline our analysis and 
show that the splitting performs well, also in comparison to more established splittings [24]. The paper concludes with an outlook in the last section.

\section{The RS-IMEX Splitting}

As mentioned before we define the splitting into stiff and non-stiff parts by linearization around the reference solution $\mathbf{w}_{(0)}$. The splitting idea has been analyzed in the context of ODEs in [41], a first extension to isentropic Euler equations was given in [42]. Similar ideas have been used before in [23].

In [41], we investigated the performance of the RS-IMEX splitting in the setting of singular perturbed ODEs and were able to obtain improved stability and accuracy results in comparison to standard splittings. This earlier work serves as a motivation to use the RS-IMEX also for the isentropic Euler equations.

Definition 1 The RS-IMEX splitting for a flux function $\mathbf{F}(\mathbf{w})$ is given by

$$
\tilde{\mathbf{F}}(\mathbf{w})=\mathbf{F}\left(\mathbf{w}_{(0)}\right)+\mathbf{F}^{\prime}\left(\mathbf{w}_{(0)}\right)\left(\mathbf{w}-\mathbf{w}_{(0)}\right), \quad \hat{\mathbf{F}}(\mathbf{w})=\mathbf{F}(\mathbf{w})-\tilde{\mathbf{F}}(\mathbf{w}),
$$

where $\mathbf{w}_{(0)}$ denotes the reference solution.

Remark 1 1. A closely related IMEX scheme was introduced by Filbet and Jin [21] for the kinetic equations with low Knudsen number. They split the collision operator into an implicit and an explicit part. As implicit part, they choose the linearization of the collision operator around the fluid dynamical limit solution, which is similar in spirit to the RS-IMEX splitting. In comparison, the $R S$-IMEX scheme splits the nonlinear flux function and the asymptotic limit PDE is the incompressible Euler equation. This needs to be solved numerically, while in the kinetic case, the background solution can be evaluated directly by computing the local Maxwellians.

2. The RS-IMEX splitting generalized work of Bispen et al. [7,8], who developed a numerical method for the shallow water equations based on a splitting due to Giraldo, Restelli and collaborators [22,23]. In that work, the asymptotic reference state was chosen to be the lake at rest, while the present schemes relies on a more general incompressible solution.

Remark 2 As a proof of concept we use the isentropic Euler equations in this work, but the RS-IMEX splitting is directly extendable to different types of equations and settings, e.g., the full Euler or Navier-Stokes equations.

A splitting into stiff and non-stiff terms has to fulfill some properties, one being that $\hat{\mathbf{F}}$ is in fact non-stiff:

Lemma 1 Let $\mathbf{n}$ be a unit normal vector. Then, the eigenvalues of $\hat{\mathbf{F}}^{\prime}(\mathbf{w}) \cdot \mathbf{n}$ are given as

$$
\widehat{\lambda}_{0}=0, \quad \widehat{\lambda}_{1}=\left(\mathbf{u}-\mathbf{u}_{(0)}\right) \cdot \mathbf{n}, \quad \widehat{\lambda}_{2}=2\left(\mathbf{u}-\mathbf{u}_{(0)}\right) \cdot \mathbf{n}
$$

for any $\mathbf{w}=(\rho, \rho \mathbf{u})^{T}$. 
In particular, one can see from La. 1 that the eigenvalues do not become singular as $\varepsilon \rightarrow 0$, i.e. the Jacobian $\hat{\mathbf{F}}^{\prime}(\mathbf{w}) \cdot \mathbf{n}$ is indeed non-stiff. In fact, a little more is true:

Corollary 1 Suppose that the asymptotic expansion (4) holds, i.e.

$$
\mathbf{u}-\mathbf{u}_{(0)}=\mathcal{O}(\varepsilon)
$$

Then the eigenvalues of the non-stiff Jacobian are in $\mathcal{O}(\varepsilon)$.

Remark 3 In the one dimensional case the difference of the eigenvectors of the non-stiff and the stiff matrices are, suitably normed, in $\mathcal{O}(\varepsilon)$. This is close to the characteristic splitting [43] where the eigenvectors of the stiff and nonstiff matrices are the same.

By construction, the stiff flux function $\tilde{\mathbf{F}}$ is linear in $\mathbf{w}$. As this part is treated implicitly, this will have a positive influence on the overall algorithm, as one only needs to solve a linear system of equations for one time step.

With Def. 1 being made, the first-order semi-discrete IMEX scheme for (8) can be written down as

$$
\frac{\mathbf{w}_{\Delta t}^{n+1}-\mathbf{w}_{\Delta t}^{n}}{\Delta t}+\nabla \cdot\left(\hat{\mathbf{F}}\left(\mathbf{w}_{\Delta t}^{n}\right)+\tilde{\mathbf{F}}\left(\mathbf{w}_{\Delta t}^{n+1}\right)\right)=0 .
$$

For the analysis to be performed, we need the following lemma, who's proof relies on the specific form of $\mathbf{w}_{(0)}$, see also (5).

Lemma 2 For $\tilde{\mathbf{F}}$ as defined in (12), there holds

$$
\nabla \cdot \tilde{\mathbf{F}}(\mathbf{w})=\nabla \cdot \mathbf{F}^{\prime}\left(\mathbf{w}_{(0)}\right) \mathbf{w}
$$

Proof The proof is straightforward computation. Using the definition of $\tilde{\mathbf{F}}$ in (12), one has to show that

$$
\nabla \cdot\left(\mathbf{F}\left(\mathbf{w}_{(0)}\right)-\mathbf{F}^{\prime}\left(\mathbf{w}_{(0)}\right) \mathbf{w}_{(0)}\right)=0
$$

Since most terms sum up to zero, the equation reduces to

$$
\nabla\left(p\left(\rho_{(0)}\right)-p^{\prime}\left(\rho_{(0)}\right) \rho_{(0)}\right)=0 .
$$

The fact that $\rho_{(0)}$ is constant, see (5), concludes the proof.

Remark 4 For $\gamma=1$, the well-known relation $\mathbf{F}(\mathbf{w})=\mathbf{F}^{\prime}(\mathbf{w}) \mathbf{w}$ holds, directly proving La. 2. For $\gamma>1$, the correction term (16) has to be accounted for.

We discretize (14) in space using a finite volume method. Therefore, the spatial domain $\Omega$ is subdivided into uniform cartesian rectangles $\Omega_{i j}$, with 
(uniform, say) cell size $\Delta x$ and unit normal vectors $\mathbf{n}_{x}$ and $\mathbf{n}_{y}$. The resulting method reads

$$
\begin{aligned}
\mathbf{w}_{i, j}^{n+1}= & \mathbf{w}_{i, j}^{n}-\frac{\Delta t}{\Delta x}\left(\widehat{\mathbf{F}}^{*}\left(\mathbf{w}_{\Delta t, i, j}^{n}, \mathbf{w}_{\Delta t, i, j+1}^{n}, \mathbf{n}_{y}\right)+\widehat{\mathbf{F}}^{*}\left(\mathbf{w}_{\Delta t, i, j}^{n}, \mathbf{w}_{\Delta t, i, j-1}^{n},-\mathbf{n}_{y}\right)\right. \\
& +\widehat{\mathbf{F}}^{*}\left(\mathbf{w}_{\Delta t, i, j}^{n}, \mathbf{w}_{\Delta t, i+1, j}^{n}, \mathbf{n}_{x}\right)+\widehat{\mathbf{F}}^{*}\left(\mathbf{w}_{\Delta t, i, j}^{n}, \mathbf{w}_{\Delta t, i-1, j}^{n},-\mathbf{n}_{x}\right) \\
& +\widetilde{\mathbf{F}}^{*}\left(\mathbf{w}_{\Delta t, i, j}^{n+1}, \mathbf{w}_{\Delta t, i, j+1}^{n+1}, \mathbf{n}_{y}\right)+\widetilde{\mathbf{F}}^{*}\left(\mathbf{w}_{\Delta t, i, j}^{n+1}, \mathbf{w}_{\Delta t, i, j-1}^{n+1},-\mathbf{n}_{y}\right) \\
& \left.+\widetilde{\mathbf{F}}^{*}\left(\mathbf{w}_{\Delta t, i, j}^{n+1}, \mathbf{w}_{\Delta t, i+1, j}^{n+1}, \mathbf{n}_{x}\right)+\widetilde{\mathbf{F}}^{*}\left(\mathbf{w}_{\Delta t, i, j}^{n+1}, \mathbf{w}_{\Delta t, i-1, j}^{n+1},-\mathbf{n}_{x}\right)\right)
\end{aligned}
$$

with a given numerical flux function $\widehat{\mathbf{F}}^{*}$ for the explicit and $\widetilde{\mathbf{F}}^{*}$ for the implicit part. For simplicity, we choose the Rusanov flux functions

$$
\begin{aligned}
\widehat{\mathbf{F}}^{*}(\mathbf{u}, \mathbf{v}, \mathbf{n}) & =\frac{\widehat{\mathbf{F}}(\mathbf{u})+\widehat{\mathbf{F}}(\mathbf{v})}{2} \mathbf{n}-\frac{1}{2} \widehat{\alpha}(\mathbf{v}-\mathbf{u}), \\
\widetilde{\mathbf{F}}^{*}(\mathbf{u}, \mathbf{v}, \mathbf{n}) & =\frac{\widetilde{\mathbf{F}}(\mathbf{u})+\widetilde{\mathbf{F}}(\mathbf{v})}{2} \mathbf{n}-\frac{1}{2} \widetilde{\alpha}(\mathbf{v}-\mathbf{u}),
\end{aligned}
$$

with constant stabilization coefficients $\hat{\alpha}$ for the explicit and $\tilde{\alpha}$ for the implicit part. Both coefficients are chosen in the order of $\varepsilon$, being motivated by the fact that $\mathbf{w}-\mathbf{w}_{(0)}=\mathcal{O}(\varepsilon)$, see also La. 1 .

\section{Asymptotic Preserving Property}

In this section, we discuss asymptotic properties of (14) and its finite volume discretization (17) coupled with certain boundary conditions. Analysis is shown in two-dimensional space; note that the results hold with direct extensions also for three-dimensional equations.

As mentioned before the equations change type for $\varepsilon \rightarrow 0$. In the setting of Euler equations this means that the equations transform to their incompressible counterpart. Thus $\varepsilon$ is a measure of the compressibility of the system, and an ideal numerical method should 'transform' to a discretization of the incompressible equations.

Definition 2 We call a numerical method asymptotically consistent (AC) if its lowest order multiscale expansion is a consistent discretization of the incompressible limit.

In Fig. 1 an illustration of the AC property is given. In order to investigate the AC property of a method, one has to compute the limit method first. Subsequently, this limit method has to be a discretization of the incompressible equations.

As mentioned in the introduction the use of a convective CFL condition is a desirable property for a numerical method in the low Mach case. In accordance to this, we define this additional quantity, guaranteeing that the method is stable under an $\varepsilon$-uniform CFL condition: 


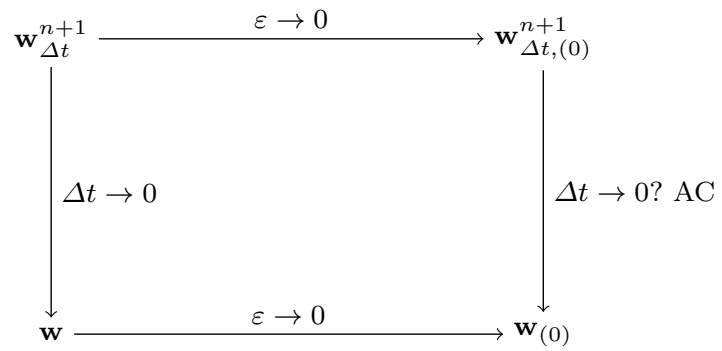

Fig. 1 Illustration of the AC property as given in [28]. If the numerical solution $\mathbf{w}_{\Delta t,(0)}^{n+1}$ converges toward $\mathbf{w}_{(0)}$ for $\Delta t \rightarrow 0$, the algorithm is asymptotically consistent (AC). Note that both $\mathbf{w}$ and $\mathbf{w}_{\Delta t}^{n+1}$ depend implicitly on $\varepsilon$ through the equation.

Definition 3 We call a numerical method asymptotically stable, see, e.g., [19], if there exists a constant $C>0$ independent of $\varepsilon$ such that the method is stable for all values of $\varepsilon \leq 1$ and for all choices of $\frac{\Delta t}{\Delta x} \leq C$.

As mentioned in the introduction, Jin introduced the asymptotic preserving property, see, e.g., [28], which is fulfilled by an asymptotically consistent scheme if the limiting numerical method is stable. In this work we prove that the method is asymptotically consistent and show that it is asymptotically stable with the help of numerical experiments. If the method is asymptotically stable, there is practically no doubt that also the limiting method is stable. Our scheme will therefore turn out to be asymptotic preserving.

\subsection{Semi-Discrete Setting: IMEX Euler}

Based on the splitting as given in (12), we consider the semi-discrete algorithm as in (14).

Lemma 3 The semi-discrete IMEX scheme (14) is asymptotically consistent if the initial data are well-prepared in the sense of (7), and mass-conserving boundary conditions are chosen.

Proof We plug the asymptotic expansion

$$
\mathbf{w}_{\Delta t}=\mathbf{w}_{\Delta t,(0)}+\varepsilon \mathbf{w}_{\Delta t,(1)}+\varepsilon^{2} \mathbf{w}_{\Delta t,(2)}+\mathcal{O}\left(\varepsilon^{3}\right)
$$

into the semi discretization (14). Because of (12) and La. 2, we obtain

$$
\mathbf{w}_{\Delta t}^{n+1}=\mathbf{w}_{\Delta t}^{n}-\Delta t \nabla \cdot\left(\mathbf{F}^{\prime}\left(\mathbf{w}_{(0)}\right) \mathbf{w}_{\Delta t}^{n+1}+\mathbf{F}\left(\mathbf{w}_{\Delta t}^{n}\right)-\mathbf{F}^{\prime}\left(\mathbf{w}_{(0)}\right) \mathbf{w}_{\Delta t}^{n}\right) .
$$

Showing that this is a consistent discretization of the incompressible Euler equations is done inductively, where the base case holds because of the wellpreparedness of the data. Then, collecting all terms of order $\varepsilon^{-2}$ in (19), we obtain

$$
0=\gamma \nabla \rho_{\Delta t,(0)}^{n+1}\left(\rho_{(0)}\right)^{\gamma-1}+\nabla\left(\rho_{\Delta t,(0)}^{n}\right)^{\gamma}-\gamma \nabla \rho_{\Delta t,(0)}^{n}\left(\rho_{(0)}\right)^{\gamma-1}
$$


Because $\nabla \rho_{\Delta t,(0)}^{n}=0$ and the fact that, in particular, $\rho_{(0)}$ is a constant in space, we get:

$$
0=\nabla \rho_{\Delta t,(0)}^{n+1},
$$

meaning that $\rho_{\Delta t,(0)}^{n+1}$ is a constant in space. Because of conservation of mass we can conclude that $\rho_{\Delta t,(0)}^{n+1}$ and $\rho_{\Delta t,(0)}^{n}$ must be the same constant and so $\rho_{\Delta t,(0)}^{n+1}$ is also constant in time. With similar arguments starting with a comparison in order $\varepsilon^{-1}$ we can conclude that $\rho_{\Delta t,(1)}^{n+1}$ is constant too.

The continuity equation to order $\varepsilon^{0}$ is given by

$$
\rho_{\Delta t,(0)}^{n+1}=\rho_{\Delta t,(0)}^{n}-\Delta t \nabla \cdot\left(\rho_{\Delta t,(0)}^{n+1} \mathbf{u}_{\Delta t,(0)}^{n+1}\right)
$$

Because $\rho_{\Delta t,(0)}^{n+1}=\rho_{\Delta t,(0)}^{n}=$ const there holds:

$$
0=\nabla \cdot \mathbf{u}_{\Delta t,(0)}^{n+1}
$$

We showed that for well-prepared initial data also $\mathbf{w}_{\Delta t}^{n}$ is well-prepared for every $n$. Making a coefficients comparison in order $\varepsilon^{0}$ we can confirm that also the discretization of the momentum equation is a consistent approximation of (8), and so for $\varepsilon \rightarrow 0$ (14) is a consistent discretization of (5).

\subsection{Fully-Discrete Setting: IMEX Euler and Finite Volume}

Previously, we have seen that the RS-IMEX splitting combined with an IMEX Euler time discretization is asymptotically consistent. The canonical next step is to show the same for the fully-discrete setting, viz for using a first order finite volume spatial discretization (17). Note that due to the structure of the RS-IMEX splitting we are able to choose both stabilization coefficients in $\mathcal{O}(\varepsilon)$. Thus taking $\varepsilon \rightarrow 0$ the numerical viscosity does not affect the lower order terms, e.g., the terms in order $\varepsilon^{i}$ with $-2 \leq i \leq 0$.

As in the analysis before, we assume that we can represent the discrete solution by

$$
\mathbf{w}_{\Delta t}=\mathbf{w}_{\Delta t,(0)}+\varepsilon \mathbf{w}_{\Delta t,(1)}+\varepsilon^{2} \mathbf{w}_{\Delta t,(2)}+\mathcal{O}\left(\varepsilon^{3}\right) .
$$

Plugging this expansion into the numerical method (17), collecting $\mathcal{O}\left(\varepsilon^{-2}\right)$ terms of the momentum equation and noting that $\rho_{(0), i, j}^{n} \equiv \rho_{(0)}$ is constant in space and time, one obtains

$$
\begin{aligned}
0=p( & \left.\rho_{(0)}\right)+p^{\prime}\left(\rho_{(0)}\right)\left(\rho_{\Delta t,(0), i, j+1}^{n+1}-\rho_{(0)}\right) \\
& -p\left(\rho_{(0)}\right)-p^{\prime}\left(\rho_{(0)}\right)\left(\rho_{\Delta t,(0), i, j-1}^{n+1}-\rho_{(0)}\right) \\
& +p\left(\rho_{\Delta t,(0), i, j+1}^{n}\right)-p\left(\rho_{(0)}\right)-p^{\prime}\left(\rho_{(0)}\right)\left(\rho_{\Delta t,(0), i, j+1}^{n}-\rho_{(0)}\right) \\
& -p\left(\rho_{\Delta t,(0), i, j-1}^{n}\right)+p\left(\rho_{(0)}\right)+p^{\prime}\left(\rho_{(0)}\right)\left(\rho_{\Delta t,(0), i, j-1}^{n+1}-\rho_{(0)}\right) .
\end{aligned}
$$


Assuming that the initial values are given in such a way that $\rho_{\Delta t,(0), i, j}^{n}$ is constant in space we obtain

$$
0=p^{\prime}\left(\rho_{(0)}\right)\left(\rho_{\Delta t,(0), i, j+1}^{n+1}-\rho_{\Delta t,(0), i, j-1}^{n+1}\right)
$$

and correspondingly

$$
0=p^{\prime}\left(\rho_{(0)}\right)\left(\rho_{\Delta t,(0), i+1, j}^{n+1}-\rho_{\Delta t,(0), i-1, j}^{n+1}\right) .
$$

Unfortunately, from this, one can not directly conclude that $\rho_{\Delta t,(0), i, j}^{n+1}$ is a constant in space. In particular, depending on the boundary conditions, checkerboard modes could occur. (See also Bispen's dissertation for a discussion of this phenomenon [7].) We note that checkerboard modes can not be precluded for periodic boundary conditions. The issue could be solved by implicit diffusion in the mass equation [7]. As we do not observe any numerical issues, we do not pursue this approach further.

Using the boundary conditions proposed by Haack, Jin and Liu in a similar setting, see [24], one can prove the AC property. More precisely, said boundary conditions operate on ghost cells and are, on the lower left boundary for example, given by

$$
\begin{aligned}
\rho_{\Delta t,-1, j}^{n} & =\rho_{\Delta t, 0, j}^{n}, & \rho_{\Delta t, i,-1}^{n} & =\rho_{\Delta t, i, 0}^{n}, \\
\rho \mathbf{u}_{\Delta t,-1, j}^{n} & =-\rho \mathbf{u}_{\Delta t, 0, j}^{n}, & \rho \mathbf{u}_{\Delta t, i,-1}^{n} & =-\rho \mathbf{u}_{\Delta t, i, 0}^{n},
\end{aligned}
$$

These boundary conditions approximate slip boundary conditions with an extra condition on $\nabla p \cdot \mathbf{n}$,

$$
\frac{\partial \rho}{\partial \mathbf{n}}=0 \quad \text { and } \quad \mathbf{u} \cdot \mathbf{n}=0 \quad \text { on } \quad \partial \Omega .
$$

To mimic well-prepared initial data as in (7), we require that the discrete initial conditions are chosen such that (note that $\mathbf{u}=(u, v)$ )

$$
\begin{aligned}
& \rho_{\Delta t, i, j}^{0}=\rho_{(0)}+\mathcal{O}\left(\varepsilon^{2}\right) \quad \text { and } \\
& u_{\Delta t, i, j+1}^{0}-u_{\Delta t, i, j-1}^{0}+v_{\Delta t, i+1, j}^{0}-v_{\Delta t, i-1, j}^{0}=\mathcal{O}(\varepsilon)+\mathcal{O}\left(\Delta x^{q}\right),
\end{aligned}
$$

holds for a $q>1$. Furthermore we assume that the reference solution was computed by a suitable numerical method. This especially means that the velocity is divergence free in a discrete sense, similar to the well-prepared initial conditions. Using these assumptions we obtain the main lemma of this section.

Lemma 4 The limiting numerical method is a consistent discretization of the limiting Euler equations, if the used reference solution is computed by a suitable numerical method, the initial conditions are well-prepared as in (28) and the boundary conditions are given as in (26). 
Proof Due to (24)-(25), and the choice of boundary conditions, we can directly conclude that $\rho_{\Delta t,(0), i, j}^{n+1}$ is constant in space. It is therefore denoted by $\rho_{\Delta t,(0)}^{n+1}$. Next we consider the $\mathcal{O}(1)$ terms of the conservation of mass equation. This results in

$$
\begin{aligned}
\rho_{\Delta t,(0)}^{n+1}=\rho_{(0)}{ }^{n}-\frac{\Delta t}{\Delta x} \frac{1}{2} & \left(\rho_{\Delta t,(0)}^{n+1} u_{\Delta t,(0), i, j+1}^{n+1}-\rho_{\Delta t,(0)}^{n+1} u_{\Delta t,(0), i, j-1}^{n+1}\right. \\
& \left.+\rho_{\Delta t,(0)}^{n+1} v_{\Delta t,(0), i+1, j}^{n+1}-\rho_{\Delta t,(0)}^{n+1} v_{\Delta t,(0), i-1, j}^{n+1}\right) .
\end{aligned}
$$

Summing this equation over the whole domain and using the boundary condition directly leads to

$$
\rho_{\Delta t,(0)}^{n+1}=\rho_{(0)}^{n} .
$$

Thus the limiting density is constant in space and time and equals to $\rho_{(0)}$ due to the well-prepared initial conditions. Note that one can show the same result for $\rho_{(1)}$ by considering the $\mathcal{O}\left(\varepsilon^{-1}\right)$ terms and performing the same steps as before. Thus due to the initial conditions we can obtain that $\rho_{(1)}=0$. The $\mathcal{O}(1)$ terms of the conservation of mass equation reduces to

$$
0=\frac{1}{\Delta x}\left(u_{\Delta t,(0), i, j+1}^{n+1}-u_{\Delta t,(0), i, j-1}^{n+1}+v_{\Delta t,(0), i+1, j}^{n+1}-v_{\Delta t,(0), i-1, j}^{n+1}\right) .
$$

This is a consistent discretization of the divergence free constraint of the limiting partial differential equation. Finally, we have to show that the remaining $\mathcal{O}(1)$ terms of the momentum equation are consistent to the corresponding limiting equation. If one considers these equations and rearranges the terms, one obtains that the first terms are a discretization of

$$
\left(\mathbf{u}_{(0)}\right)_{t}+\nabla \cdot\left(\mathbf{u}_{(0)} \otimes \mathbf{u}_{(0)}\right)+\frac{1}{\rho_{(0)}} \nabla p_{(2)}=0
$$

which is indeed the momentum equation of the incompressible Euler equation, and the remaining terms are terms in the difference of the computed solution and the reference solution. Therefore, if the reference solution is computed in a suitable way the limiting numerical method is a consistent discretization of the limiting equation.

\section{Numerical Results}

Having analyzed the RS-IMEX splitting, this section presents numerical results for the proposed splitting. The splitting is compared against a splitting from literature, introduced by Haack, Jin and Liu in [24]. The splitting is given in the following definition; it is labeled $H J L$ in the following. 
Definition 4 Haack, Jin and Liu splitting [24] (HJL). With this splitting, the convective fluxes are split into stiff and non-stiff terms, respectively, by

$$
\widetilde{\mathbf{F}}(\mathbf{w})=\left(\begin{array}{c}
(1-\tau)(\rho \mathbf{u}) \\
\frac{a(t)}{\varepsilon^{2}} \rho
\end{array}\right) \text { and } \widehat{\mathbf{F}}(\mathbf{w})=\left(\begin{array}{c}
\tau(\rho \mathbf{u}) \\
\rho \mathbf{u} \otimes \mathbf{u}+\frac{p-a(t) \rho}{\varepsilon^{2}}
\end{array}\right) .
$$

The particular choice of $\tau$ and $a(t)$ is given by

$$
0<\tau<1, \tau \in \mathcal{O}\left(\varepsilon^{2}\right) \quad \text { and } \quad a(t)=\min _{\mathbf{x}} p^{\prime}(\rho(x, t)) .
$$

The splitting is hyperbolic and the eigenvalues of $\widehat{\mathbf{F}}$ are in magnitude independent of $\varepsilon$. Also $\widetilde{\mathbf{F}}$ is a linear function, if $a(t)$ is handled explicitly, i.e., evaluated at time $t^{n}$. Note that this splitting is well tested. In [24] Haack, Jin and Liu proved that the splitting is asymptotically consistent and uniformly stable under a CFL restriction independent of $\varepsilon$. We note that the authors in [24] did not directly apply their splitting in form (14), but restate the equations in the semi-discrete setting to obtain an additional pressure equation, which is the only equation that is solved for implicitly in their algorithm.

In the following we consider two different examples and compute the $L^{1}$ error of the first momentum component. This is sufficient since both momentum components behave very similar and the density is close to a constant.

\subsection{Periodic Flow}

Inspired by Degond and Tang [20] (see also [24]) we compute the so called periodic flow example.

Definition 5 The periodic flow example is given on $\Omega=[0,1] \times[0,1]$ with periodic initial conditions

$$
\begin{aligned}
& \rho(x, y, 0)=1+\varepsilon^{2} \sin ^{2}(2 \pi(x+y)) \\
& \mathbf{u}(x, y, 0)=\left(\begin{array}{l}
\sin (2 \pi(x-y)) \\
\sin (2 \pi(x-y))
\end{array}\right)
\end{aligned}
$$

periodic boundary conditions and $\gamma=2$. The initial conditions are illustrated in Fig. 2 .

The error is computed with the aid of a highly resolved solution, being computed on a finer grid with HJL splitting and a higher-order method.

Both numerical methods are computed with the same solver and for both, moderate stabilization coefficients are used, namely $\hat{\alpha}=2 \varepsilon$ and $\tilde{\alpha}=\varepsilon$ for the RS-IMEX and $\hat{\alpha}=2$ and $\tilde{\alpha}=1$ for the HJL splitting. The reference solution is computed with a corresponding semi-implicit finite volume method where the convective term is treated explicitly and the divergence free constraint is stabilized with pressure. We use stabilization coefficients $\hat{\alpha}=\tilde{\alpha}=2$. Finally we choose $\tau=\varepsilon^{2}$ for $\varepsilon<1$ and $\tau=0.5$ for $\varepsilon=1$. 

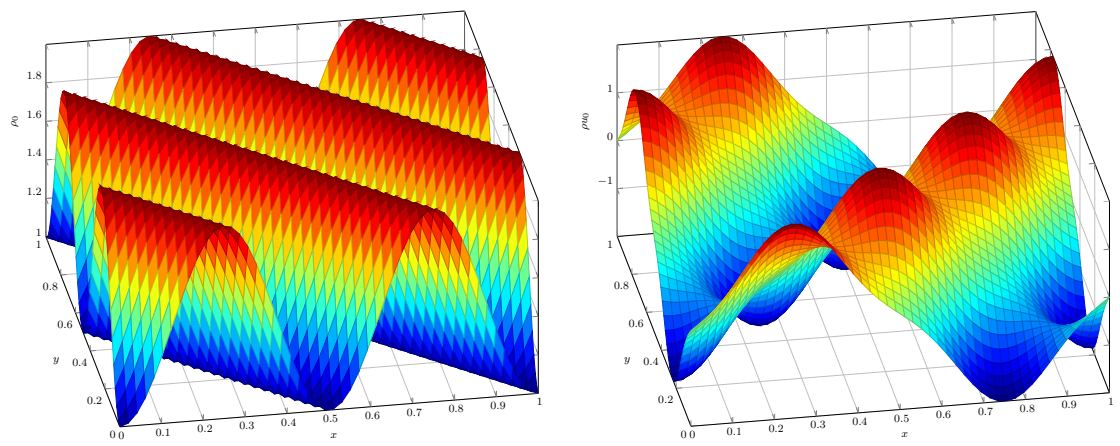

Fig. 2 Initial conditions for the periodic flow example for $\varepsilon=1$ : Left: $\rho$ and right: $\rho u$.

Using a grid cell size ranging from $\Delta x=2^{-2}$ to $\Delta x=2^{-7}$ we chose for all values of $\varepsilon$, viz $\varepsilon=10^{-i}$ with $i=0 \ldots 4$, the same relation between the cell size and the temporal step size, namely

$$
\frac{\Delta t}{\Delta x}=0.25
$$

Based on the initial conditions, this corresponds to a global convective CFL number of also 0.25 .

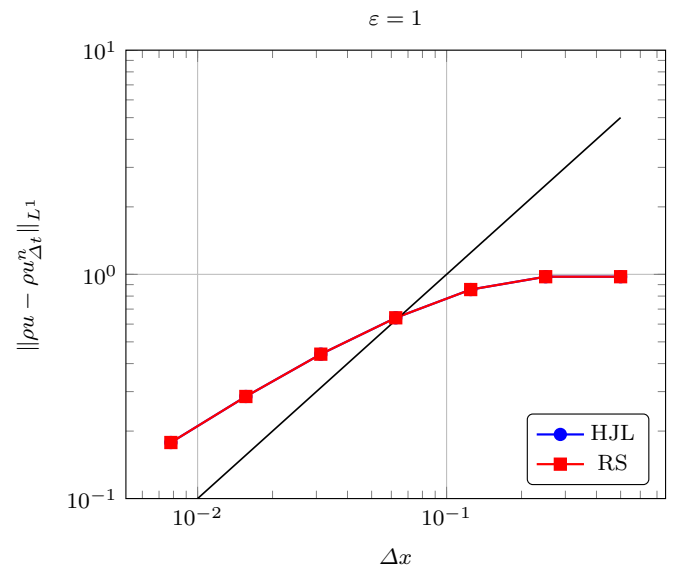

Fig. 3 Periodic flow: Comparison of the RS-IMEX with the HJL splitting for large Mach number $\varepsilon=10^{0}$. The plot shows the $L^{1}$-error in the first momentum component of the computed solution with a highly resolved numerical solution. HJL and RS lie on top of each other. Black line indicates first order convergence.

Starting with large values for $\varepsilon$ demonstrates the performance of the methods in the fully compressible regime. In Fig. 3 one can clearly see that both our newly developed method and the classical HJL method perform equally 


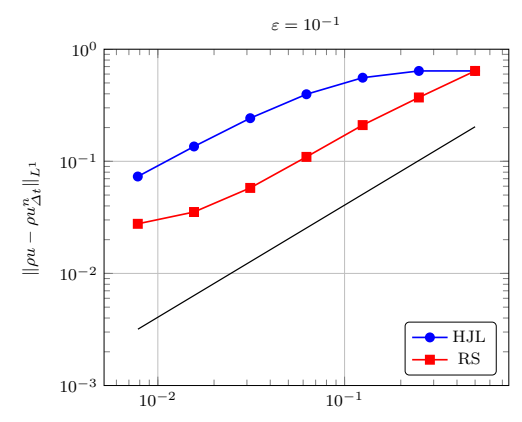

$\Delta x$
$=10^{-3}$

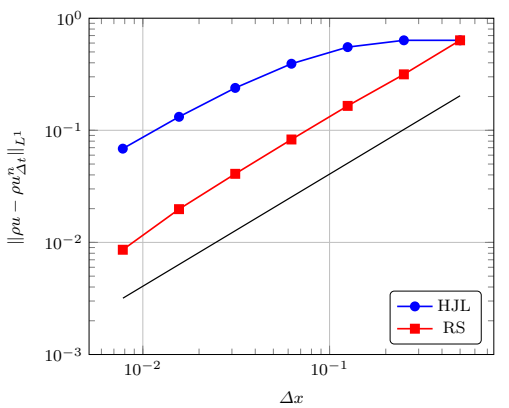

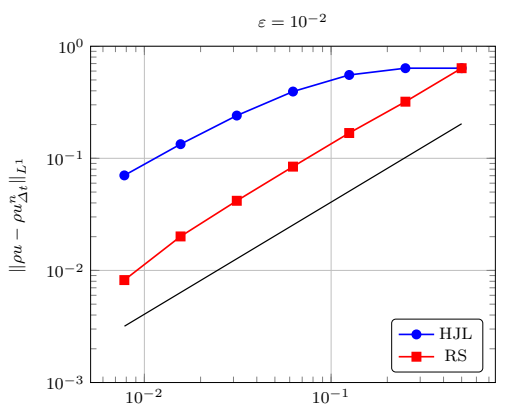

$\Delta x$

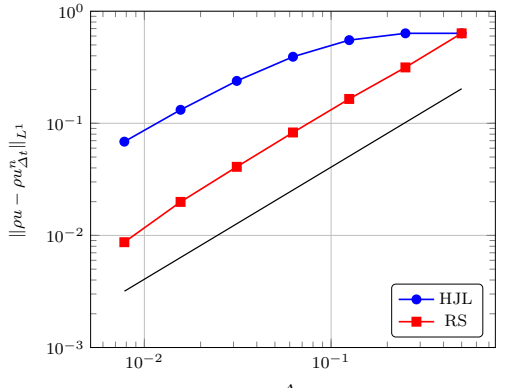

Fig. 4 Periodic flow: Comparison of the RS-IMEX with the HJL splitting for Mach numbers $\varepsilon=10^{-1}$ to $10^{-4}$. The plots show the $L^{1}$-error in the first momentum component of the computed solution with a highly resolved numerical solution. Black line indicates first order convergence.

well for $\varepsilon=1$. This is not surprising, as for $\varepsilon=1$, the solutions are not yet in the asymptotic regime.

In Fig. 4, numerical results are shown for $\varepsilon=10^{-1}$ to $10^{-4}$. Beginning from $\varepsilon=10^{-1}$, one obtains improved accuracy for the RS-IMEX splitting. Both splittings converge with the desired order. In all settings it can be clearly seen that both splittings have the same initial error on the coarse grid, but RSIMEX gives a better convergence than the HJL splitting. Ultimately, there is a factor of about 10 between both errors. In order to achieve the same error, the algorithm involving the RS-IMEX splitting needs to solve for both compressible and incompressible solution, but on a significantly coarser grid. Overall, this yields substantial savings.

From the convergence figures one can also see that both methods are AC: The smaller $\varepsilon_{1}$ and $\varepsilon_{2}$, the more similar the corresponding error plots are.

\subsection{Traveling Vortex}

As a second example, we consider the traveling vortex example used by Bispen et al. in [8]. 

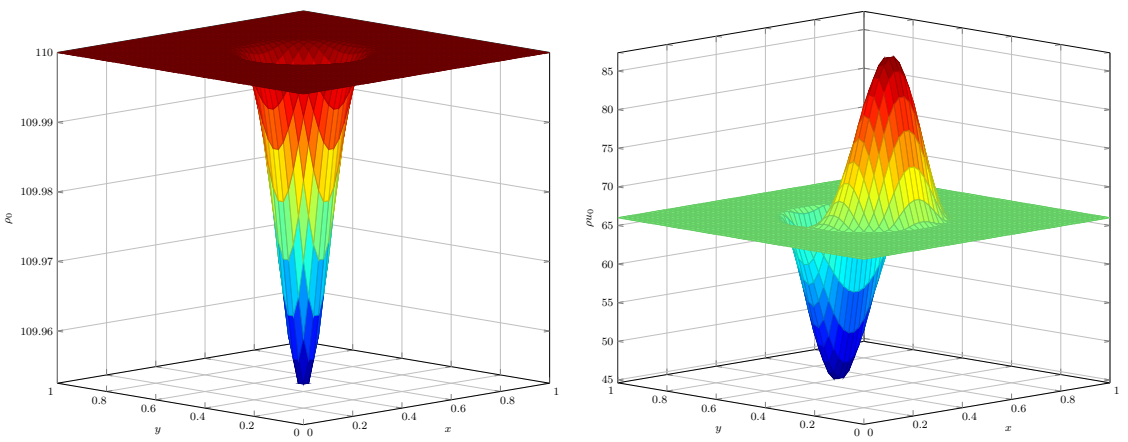

Fig. 5 Initial conditions for the traveling vortex example for $\varepsilon=1$ : Left: $\rho$ and right: $\rho u$.

Definition 6 The traveling vortex example is given on $\Omega=[0,1] \times[0,1]$ with periodic initial conditions

$$
\begin{aligned}
& \rho(x, y, 0)=110+\varepsilon^{2}\left(\frac{1.5}{4 \pi}\right)^{2} \delta\left(r_{c}\right)\left(k\left(r_{c}\right)-k(\pi)\right) \\
& \mathbf{u}(x, y, 0)=\left(\begin{array}{c}
0.6 \\
0
\end{array}\right)+1.5\left(1+\cos \left(r_{c}\right)\right) \delta\left(r_{c}\right)\left(\begin{array}{c}
(0.5-y) \\
(x-0.5)
\end{array}\right) .
\end{aligned}
$$

where

$$
\begin{aligned}
k(r) & :=2 \cos (r)+2 r \sin (r)+\frac{1}{8} \cos (2 r)+\frac{1}{4} r \sin (2 r)+\frac{3}{4} r^{2}, \\
r_{c} & :=4 \pi \sqrt{(x-0.5)^{2}+(y-0.5)^{2}}, \\
\delta(r) & := \begin{cases}1 & r<\pi \\
0 & \text { otherwise }\end{cases}
\end{aligned}
$$

Boundary conditions are chosen to be periodic (unlike in [8]), and $p(\rho)=\frac{1}{2} \rho^{2}$. The initial conditions are illustrated in Fig. 5.

Note that for this example an exact solution is available:

$$
\rho(x, y, t)=\rho(x-0.6 t, y, 0), \quad \mathbf{u}(x, y, t)=\mathbf{u}(x-0.6 t, y, 0) .
$$

We compute the results in a similar setting as before. This means we compute on the same grids and the same values of $\varepsilon$. Again we use for both methods the same relation between the temporal step size and the cell size, namely

$$
\frac{\Delta t}{\Delta x}=0.05
$$

Based on the initial conditions, this corresponds to a global convective CFL number of approximately 0.04 . The setting is the same as before, with the exception that the stabilization coefficients for the computation of the incompressible solutions are chosen to be $\hat{\alpha}=0$ and $\tilde{\alpha}=2$. 
Results in this example are given in Figs. 6-7, they are similar to those of the previous example. Again, for $\varepsilon=1$, both methods compute similarly accurate results, but the RS-IMEX splittings takes twice the work. For $\varepsilon \ll 1$ the RS-IMEX method performs much better. While the HJL splitting needs some steps to hit the right convergence order, the RS-IMEX splitting starts with the correct order after a few steps, leading to a more accurate solution. This then also justifies the higher effort due to the computation of the incompressible solution. There seems to be a slight superconvergence of the RS splitting, however, we think this is only an artefact that diminishes under grid refinement.

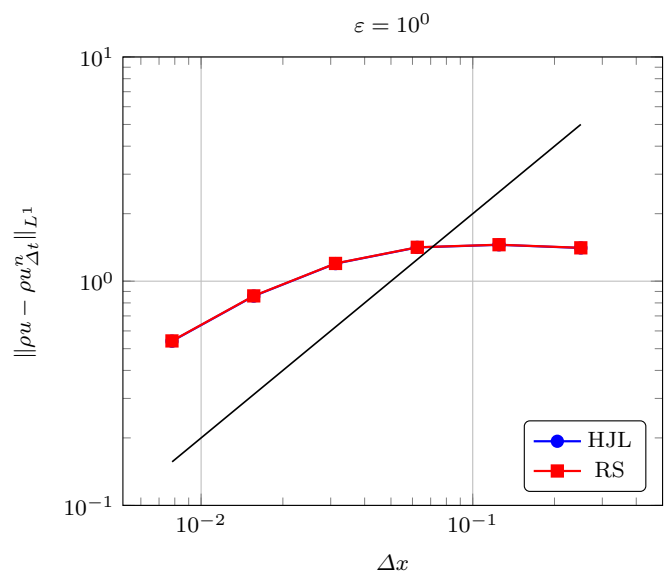

Fig. 6 Traveling vortex: Comparison of the RS-IMEX with the HJL splitting for large Mach number $\varepsilon=10^{0}$. The plot shows the $L^{1}$-error in the first momentum component of the computed solution with a highly resolved numerical solution. HJL and RS lie on top of each other. Black line indicates first order convergence.

Note, in addition, that due to the stiffness of the resulting linear system, the linear solver needs much more time to solve the compressible part than the incompressible one. Thus, for small values of $\varepsilon$, solving the incompressible part is negligible in terms of computational costs. To verify this we compare the computational costs for the implemented solver in the next subsection.

\subsection{Computational cost}

In the previous two subsections we observed that the RS-IMEX splitting delivers a better error for the same mesh size and small values of $\varepsilon$ compared to the HJL splitting. This happens with the additional effort in solving the incompressible limit equation. To make a fair comparison, one has therefore to also report computational times. We implemented a solver with the help of Netgen [39] and PETSc [4-6] to solve both the RS-IMEX and the HJL splitting. The basic solver was written for the HJL splitting and then extended by 

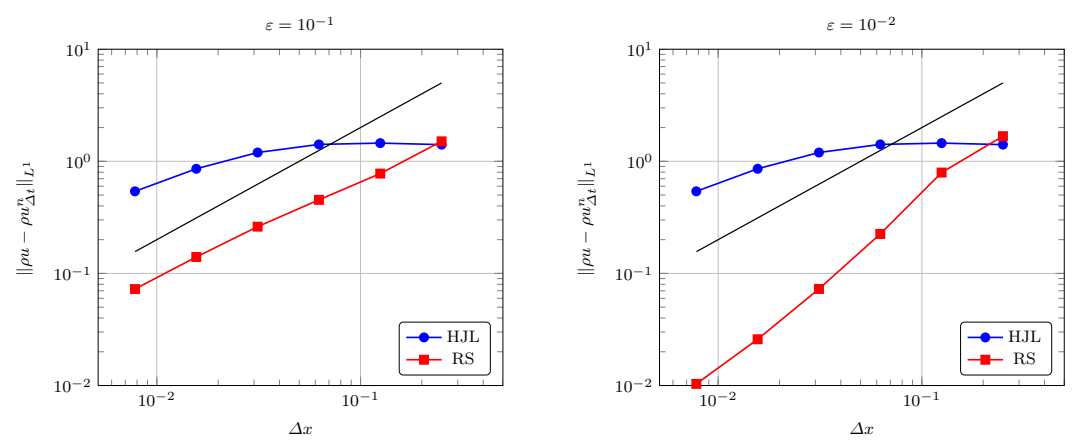

$\Delta x$
$=10^{-4}$
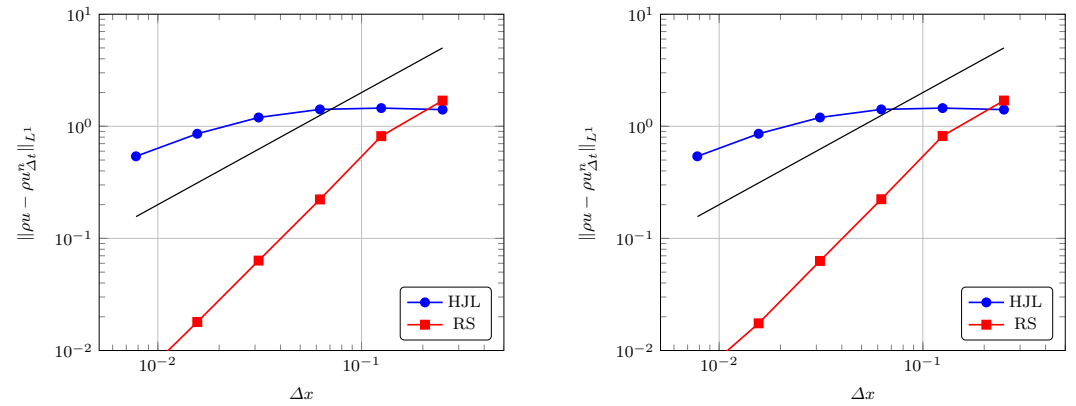

Fig. 7 Traveling vortex: Comparison of the RS-IMEX with the HJL splitting for Mach numbers $\varepsilon=10^{-1}$ to $10^{-4}$. The plots show the $L^{1}$-error in the first momentum component of the computed solution with a highly resolved numerical solution. Black line indicates first order convergence.

an incompressible solver. We only use basic techniques to solve the implicit system, therefore the solver is not optimized in any sense. Note that the system of equations gets harder to solve if one reduces the mesh size $\Delta x$ or $\varepsilon$. This plays an important role in choosing the right residual threshold value to stop the solver. We believe that one can, especially for the low Mach case, reduce the computational costs significantly by optimization. The basic solver was designed as a proof of concept.

In Tables 1 and 2 we see the computational costs of both methods to compute a similar error for different values of $\varepsilon$. For large values of $\varepsilon$ we can see that the HJL splitting performs much better, since the RS-IMEX splitting needs to solve more equations. Therefore for large values of $\varepsilon$ this additional effort is not justified. For small values of $\varepsilon$ the RS-IMEX splitting reduces the computational costs significantly since one uses a coarser grid to get the same error. For the traveling vortex example difference is much larger due to the superconvergence we obtained in the beginning. 


\begin{tabular}{|c||c||c|c|c|c|}
\hline$\varepsilon$ & method & $\Delta x$ & ne & $L^{1}$-error & time in s \\
\hline $10^{0}$ & $H J L$ & $7.8125 \cdot 10^{-3}$ & $\left(2^{7}\right)^{2}$ & 0.1778 & $7.40 \cdot 10^{1}$ \\
& $R S$-IMEX & $7.8125 \cdot 10^{-3}$ & $\left(2^{7}\right)^{2}$ & 0.1778 & $4.52 \cdot 10^{2}$ \\
\hline \multirow{2}{*}{$10^{-1}$} & $H J L$ & $7.8125 \cdot 10^{-3}$ & $\left(2^{7}\right)^{2}$ & 0.0732 & $1.95 \cdot 10^{2}$ \\
& $R S-I M E X$ & $3.125 \cdot 10^{-2}$ & $\left(2^{5}\right)^{2}$ & 0.0579 & $5.02 \cdot 10^{0}$ \\
\hline \multirow{2}{*}{$10^{-2}$} & $H J L$ & $7.8125 \cdot 10^{-3}$ & $\left(2^{7}\right)^{2}$ & 0.0704 & $1.45 \cdot 10^{3}$ \\
& $R S-I M E X$ & $3.125 \cdot 10^{-2}$ & $\left(2^{5}\right)^{2}$ & 0.0418 & $1.54 \cdot 10^{1}$ \\
\hline \multirow{2}{*}{$10^{-3}$} & $H J L$ & $7.8125 \cdot 10^{-3}$ & $\left(2^{7}\right)^{2}$ & 0.0686 & $2.04 \cdot 10^{3}$ \\
& $R S-I M E X$ & $3.125 \cdot 10^{-2}$ & $\left(2^{5}\right)^{2}$ & 0.0409 & $3.86 \cdot 10^{1}$ \\
\hline \multirow{2}{*}{$10^{-4}$} & $H J L$ & $7.8125 \cdot 10^{-3}$ & $\left(2^{5}\right)^{2}$ & 0.0686 & $4.20 \cdot 10^{3}$ \\
& $R S-I M E X$ & $3.125 \cdot 10^{-2}$ & $\left(2^{5}\right)^{2}$ & 0.0409 & $4.13 \cdot 10^{1}$ \\
\hline
\end{tabular}

Table 1 Periodic flow example: Comparison of computational time to obtain a similar $L^{1}$-error of the first momentum component for the $H J L$ splitting and $R S-I M E X$ splitting.

\begin{tabular}{|c||c||c|c|c|c|}
\hline$\varepsilon$ & method & $\Delta x$ & ne & $L^{1}$-error & time in s \\
\hline \multirow{2}{*}{$10^{0}$} & $H J L$ & $7.8125 \cdot 10^{-3}$ & $\left(2^{7}\right)^{2}$ & 0.5401 & $3.24 \cdot 10^{2}$ \\
& $R S-I M E X$ & $7.8125 \cdot 10^{-3}$ & $\left(2^{7}\right)^{2}$ & 0.5415 & $1.08 \cdot 10^{4}$ \\
\hline \multirow{2}{*}{$10^{-1}$} & $H J L$ & $7.8125 \cdot 10^{-3}$ & $\left(2^{7}\right)^{2}$ & 0.5398 & $2.02 \cdot 10^{3}$ \\
& $R S-I M E X$ & $6.25 \cdot 10^{-2}$ & $\left(2^{4}\right)^{2}$ & 0.4530 & $5.02 \cdot 10^{0}$ \\
\hline \multirow{2}{*}{$10^{-2}$} & $H J L$ & $7.8125 \cdot 10^{-3}$ & $\left(2^{7}\right)^{2}$ & 0.5398 & $1.53 \cdot 10^{4}$ \\
& $R S-I M E X$ & $6.25 \cdot 10^{-2}$ & $\left(2^{4}\right)^{2}$ & 0.2249 & $8.81 \cdot 10^{0}$ \\
\hline \multirow{2}{*}{$10^{-3}$} & $H J L$ & $7.8125 \cdot 10^{-3}$ & $\left(2^{7}\right)^{2}$ & 0.5398 & $2.07 \cdot 10^{4}$ \\
& $R S-I M E X$ & $6.25 \cdot 10^{-2}$ & $\left(2^{4}\right)^{2}$ & 0.2227 & $3.15 \cdot 10^{1}$ \\
\hline \multirow{2}{*}{$10^{-4}$} & $H J L$ & $7.8125 \cdot 10^{-3}$ & $\left(2^{7}\right)^{2}$ & 0.5397 & $9.77 \cdot 10^{4}$ \\
& $R S-I M E X$ & $6.25 \cdot 10^{-2}$ & $\left(2^{4}\right)^{2}$ & 0.2236 & $7.89 \cdot 10^{1}$ \\
\hline
\end{tabular}

Table 2 Traveling vortex example: Comparison of computational time to obtain a similar $L^{1}$-error of the first momentum component for the $H J L$ splitting and $R S-I M E X$ splitting.

\section{Conclusion and Outlook}

We have presented a new IMEX flux splitting for two-dimensional, low Mach number, isentropic compressible Euler equations, as a prototype for stiff hyperbolic systems of conservation laws. The stiff part is the linearization of the flux around the asymptotic reference solution (in this case the incompressible solution).

We have proven that the new scheme is asymptotically consistent, and our numerical experiments indicate that it is stable under a convective CFL condition, and for all values of $\varepsilon$. Hence the scheme may be called asymptotic preserving. 
For two well-known test cases, the new splitting gives smaller errors than Haack, Jin and Liu's recent splitting on the same mesh (when compared within the same IMEX code). For small $\varepsilon$, similar errors are obtained within less computational time.

Several extensions of the new method are important: high-order discontinuous Galerkin methods [12-16] and IMEX Runge-Kutta integrators [2,30] to increase the efficiency and accuracy, development and implementation for more complex equations, as well as analytical proofs of asymptotic stability.

\section{Acknowledgements}

The first author has been partially supported by DFG project NO 361/3-3 (German Science Foundation).

The authors would like to thank Arun K.R., Georgij Bispen, Rupert Klein, Mária Lukáčová-Medvid'ová, Claus-Dieter Munz and Hamed Zakerzadeh for the discussions and collaborations leading to the RS-IMEX approach.

\section{References}

1. Anderson, J.D.: Fundamentals of Aerodynamics, $3^{\text {rd }}$ edn. McGraw-Hill New York (2001)

2. Ascher, U.M., Ruuth, S., Spiteri, R.: Implicit-explicit Runge-Kutta methods for timedependent partial differential equations. Applied Numerical Mathematics 25, 151-167 (1997)

3. Ascher, U.M., Ruuth, S., Wetton, B.: Implicit-explicit methods for time-dependent partial differential equations. SIAM Journal on Numerical Analysis 32, 797-823 (1995)

4. Balay, S., Brown, J., Buschelman, K., Eijkhout, V., Gropp, W.D., Kaushik, D., Knepley, M.G., McInnes, L.C., Smith, B.F., Zhang, H.: PETSc users manual. Tech. Rep. ANL95/11 - Revision 3.1, Argonne National Laboratory (2010)

5. Balay, S., Brown, J., Buschelman, K., Gropp, W.D., Kaushik, D., Knepley, M.G., McInnes, L.C., Smith, B.F., Zhang, H.: PETSc Web page (2011). Http://www.mcs.anl.gov/petsc

6. Balay, S., Gropp, W.D., McInnes, L.C., Smith, B.F.: Efficient management of parallelism in object oriented numerical software libraries. In: E. Arge, A.M. Bruaset, H.P. Langtangen (eds.) Modern Software Tools in Scientific Computing, pp. 163-202. Birkhäuser Press Boston (1997)

7. Bispen, G.: IMEX finite volume methods for the shallow water equations. Ph.D. thesis, Johannes Gutenberg-Universität (2015)

8. Bispen, G., Arun, K., Lukáčová-Medvid'ová, M., Noelle, S.: IMEX large time step finite volume methods for low Froude number shallow water flows. Communications in Computational Physics 16, 307-347 (2014)

9. Boscarino, S.: Error analysis of IMEX Runge-Kutta methods derived from differentialalgebraic systems. SIAM Journal on Numerical Analysis 45, 1600-1621 (2007)

10. Boscarino, S.: On an accurate third order implicit-explicit Runge-Kutta method for stiff problems. Applied Numerical Mathematics 59, 1515-1528 (2009)

11. Boscarino, S., Pareschi, L., Russo, G.: Implicit-explicit Runge-Kutta schemes for hyperbolic systems and kinetic equations in the diffusion limit. SIAM Journal on Scientific Computing 35(1), A22-A51 (2013)

12. Cockburn, B., Hou, S., Shu, C.W.: The Runge-Kutta local projection discontinuous Galerkin finite element method for conservation laws IV: The multidimensional case. Mathematics of Computation 54, 545-581 (1990) 
13. Cockburn, B., Lin, S.Y.: TVB Runge-Kutta local projection discontinuous Galerkin finite element method for conservation laws III: One dimensional systems. Journal of Computational Physics 84, 90-113 (1989)

14. Cockburn, B., Shu, C.W.: TVB Runge-Kutta local projection discontinuous Galerkin finite element method for conservation laws II: General framework. Mathematics of Computation 52, 411-435 (1988)

15. Cockburn, B., Shu, C.W.: The Runge-Kutta local projection $p^{1}$-discontinuous Galerkin finite element method for scalar conservation laws. RAIRO Mathematical modelling and numerical analysis 25, 337-361 (1991)

16. Cockburn, B., Shu, C.W.: The Runge-Kutta discontinuous Galerkin Method for conservation laws V: Multidimensional Systems. Mathematics of Computation 141, 199-224 (1998)

17. Cordier, F., Degond, P., Kumbaro, A.: An asymptotic-preserving all-speed scheme for the Euler and Navier-Stokes equations. Journal of Computational Physics 231, 5685$5704(2012)$

18. Crouzeix, M.: Une méthode multipas implicite-explicite pour l'approximation des équations d'évolution paraboliques. Numerische Mathematik 35(3), 257-276 (1980)

19. Degond, P., Jin, S., Liu, J.G.: Mach-number uniform asymptotic-preserving gauge schemes for compressible flows. Bulletin-Institute of Mathematics Academica Sinicia 2(4), 851 (2007)

20. Degond, P., Tang, M.: All speed scheme for the low Mach number limit of the isentropic Euler equation. Communications in Computational Physics 10, 1-31 (2011)

21. Filbet, F., Jin, S.: A class of asymptotic-preserving schemes for kinetic equations and related problems with stiff sources. Journal of Computational Physics 229(20), 76257648 (2010)

22. Giraldo, F., Restelli, M., Läuter, M.: Semi-implicit formulations of the Navier-Stokes equations: Application to nonhydrostatic atmospheric modeling. SIAM Journal on Scientific Computing 32(6), 3394-3425 (2010)

23. Giraldo, F.X., Restelli, M.: High-order semi-implicit time-integrators for a triangular discontinuous Galerkin oceanic shallow water model. International Journal for Numerical Methods in Fluids 63(9), 1077-1102 (2010)

24. Haack, J., Jin, S., Liu, J.G.: An all-speed asymptotic-preserving method for the isentropic Euler and Navier-Stokes equations. Communications in Computational Physics 12, 955-980 (2012)

25. Hairer, E., Wanner, G.: Solving Ordinary Differential Equations II. Springer Series in Computational Mathematics (1991)

26. Hundsdorfer, W., Ruuth, S.J.: IMEX extensions of linear multistep methods with general monotonicity and boundedness properties. Journal of Computational Physics 225(2), 2016-2042 (2007)

27. Jin, S.: Efficient asymptotic-preserving (AP) schemes for some multiscale kinetic equations. SIAM Journal on Scientific Computing 21, 441-454 (1999)

28. Jin, S.: Asymptotic preserving (AP) schemes for multiscale kinetic and hyperbolic equations: A review. Rivista di Matematica della Universita Parma 3, 177-216 (2012)

29. Jin, S., Pareschi, L., Toscani, G.: Diffusive relaxation schemes for multiscale discretevelocity kinetic equations. SIAM Journal on Numerical Analysis 35, 2405-2439 (1998)

30. Kennedy, C.A., Carpenter, M.H.: Additive Runge-Kutta schemes for convectiondiffusion-reaction equations. Applied Numerical Mathematics 44, 139-181 (2003)

31. Klainerman, S., Majda, A.: Singular limits of quasilinear hyperbolic systems with large parameters and the incompressible limit of compressible fluids. Communications on Pure and Applied Mathematics 34, 481-524 (1981)

32. Klein, R.: Semi-implicit extension of a Godunov-type scheme based on low Mach number asymptotics I: One-dimensional flow. Journal of Computational Physics 121, 213-237 (1995)

33. Kröner, D.: Numerical schemes for conservation laws. Wiley Teubner (1997)

34. Kuepper, K., Frank, M., Jin, S.: An asymptotic preserving 2-d staggered grid method for multiscale transport equations. SIAM Journal on Numerical Analysis 54, 440-461 (2016) 
35. Müller, A., Behrens, J., Giraldo, F., Wirth, V.: Comparison between adaptive and uniform discontinuous Galerkin simulations in dry 2d bubble experiments. Journal of Computational Physics 235, 371-393 (2013)

36. Noelle, S., Bispen, G., Arun, K., Lukáčová-Medvid'ová, M., Munz, C.D.: A weakly asymptotic preserving low Mach number scheme for the Euler equations of gas dynamics. SIAM Journal of Scientific Computing 36, B989-B1024 (2014)

37. Pareschi, L., Russo, G.: Implicit-explicit runge-kutta schemes and applications to hyperbolic systems with relaxation. Journal of Scientific Computing 25(1-2), 129-155 (2005)

38. Restelli, M.: Semi-lagrangian and semi-implicit discontinuous Galerkin methods for atmospheric modeling applications. PhD thesis Politecnico di Milano (2007)

39. Schöberl, J.: Netgen - an advancing front $2 \mathrm{~d} / 3 \mathrm{~d}$-mesh generator based on abstract rules. Computing and Visualization in Science 1, 41-52 (1997)

40. Schochet, S.: Fast singular limits of hyperbolic PDEs. Journal of Differential Equations 114(2), 476-512 (1994)

41. Schütz, J., Kaiser, K.: A new stable splitting for singularly perturbed ODEs. Applied Numerical Mathematics 107, 18-33 (2016)

42. Schütz, J., Kaiser, K., Noelle, S.: The RS-IMEX splitting for the isentropic Euler equations. In: S. Elgeti, J.W. Simon (eds.) Conference Proceedings of the YIC GACM 2015. Publication Server of RWTH Aachen University (2015). DOI urn:nbn:de:hbz:82-rwth-2015-039806. URL https://publications.rwthaachen.de/record/480970/files/ProceedingsYIC-GACM-ACCES.pdf

43. Schütz, J., Noelle, S.: Flux splitting for stiff equations: A notion on stability. Journal of Scientific Computing 64(2), 522-540 (2015)

44. Wesseling, P.: Principles of Computational Fluid Dynamics, Springer Series in Computational Mechanics, vol. 29. Springer Verlag (2001)

45. Yelash, L., Müller, A., Lukáčová-Medvid'ová, M., Giraldo, F.X., Wirth, V.: Adaptive discontinuous evolution Galerkin method for dry atmospheric flow. Journal of Computational Physics 268, 106-133 (2014)

46. Yong, W.A.: A note on the zero Mach number limit of compressible Euler equations. Proceedings of the American Mathematical Society 133(10), 3079-3085 (2005)

47. Zakerzadeh, H., Noelle, S.: A note on the stability of implicit-explicit flux splittings for stiff hyperbolic systems. IGPM Preprint Nr. 449 (2016) 